\title{
El sitio arqueológico de Los Padres en Zapopan: propuesta de intervención y divulgación para su conservación
}

\author{
Laura Patricia Montoya Barragán \\ Daniel Rodríguez Medina \\ Universidad de Guadalajara
}

\section{Resumen}

El sitio arqueológico de Los Padres, así como los sitios del municipio de Zapopan, son representativos de las culturas del Valle de Atemajac. Lamentablemente, el crecimiento acelerado de la población a las periferias de la ciudad, dejó esta zona inmersa dentro de la mancha urbana, convirtiéndose en un predio baldío y por consecuencia, en un área insegura para los vecinos de la zona. Debido a esto, es necesaria la intervención en el sitio y la divulgación de la información que se tiene sobre este, con el fin de generar un interés por parte de la sociedad y por lo tanto, lograr su conservación.

Palabras clave: Arqueología, Los Padres, Valle de Atemajac, parque arqueológico, realidad aumentada.

\section{Abstract}

The archaeological site of Los Padres, as well as all the sites of the municipality of Zapopan, are representative of the cultures of the Atemajac Valley. Unfortunately, the rapid growth of the population to the peripheries of the city, left this area within the urban stain becoming a wasteland and consequently, an area of insecurity for neighbors. That's why it is urgently needed an intervention on this site and divulgation of information about it, creating an interest in the society and, consequently, achieve its conservation.

Key words: Archeology, archaeological park, augmented reality. 


\section{Introducción}

Por mucho tiempo se pensó que la región Occidente de Mesoamérica carecía de culturas prehispánicas y de vestigios arqueológicos, sin embargo, hoy se sabe que no fue así. Tan solo en el municipio de Zapopan en el estado de Jalisco, existen nueve sitios arqueológicos registrados por el Instituto Nacional de Antropología e Historia (INAH), y que actualmente se encuentran dentro de la mancha urbana del Área Metropolitana de Guadalajara (AMG).

Los polígonos correspondientes a los nueve sitios arqueológicos de Zapopan fueron declarados áreas de protección del patrimonio histórico en los planes parciales de desarrollo urbano, sin embargo, aún permanecen bajo la maleza (a excepción del sitio arqueológico El Iztépete, también conocido como El Ixtépete), y con el paso del tiempo, fueron confinados dentro de la mancha urbana esperando ser estudiados y admirados por los habitantes del AMG.

Las investigaciones sobre el patrimonio arqueológico del Valle de Atemajac son de difícil acceso debido a que muchas de ellas no son publicadas y se encuentran únicamente en propiedad del INAH. Actualmente, los nueve sitios son focos de inseguridad para los vecinos de las zonas arqueológicas al convertirse en extensos predios baldíos carentes de alumbrado público que recolectan basura en la malla perimetral del sitio.

Los sitios se encuentran en lamentable estado de conservación, incluyendo al sitio arqueológico El Iztépete, debido a la falta de divulgación, el conocimiento general en la población acerca de los sitios ubicados en el Valle de Atemajac es prácticamente nula, generando vandalismo, saqueo y destrucción de los vestigios arqueológicos.

\section{Objetivos}

El presente documento analiza el estado actual de conservación de uno de los nueve sitios arqueológicos, su contexto y el papel de la población en el tema de la protección del patrimonio edificado, con el fin de promover la divulgación de la información científica al público en general. Se presenta un criterio de intervención que pueda aplicarse no solo a Los Padres, sino a todos los sitios arqueológicos los cuales su estado de conservación se encuentre en la misma situación. Con el uso de herramientas tecnológicas para divulgación de la información, se podrá lograr un mejor entendimiento de las estructuras prehispánicas de esta área mesoamericana y por consecuencia, una mayor participación de la sociedad en el tema de la conservación del patrimonio edificado. 


\section{Metodología}

Se ha hablado sobre la importancia de conservar el patrimonio edificado para las generaciones futuras, sin embargo, este tema se ha mantenido únicamente dentro de la comunidad científica dejando ausente la participación de la sociedad en general en la conservación del patrimonio.

El patrimonio arqueológico en el municipio Zapopan ha permanecido en estado de abandono. Debido a ello, en este documento se presenta una propuesta de intervención conceptual y divulgación del patrimonio arqueológico del Valle de Atemajac.

Se decidió analizar el sitio arqueológico de Los Padres en el municipio de Zapopan, Jalisco, debido a que es uno de los sitios que presenta una mayor problemática en cuanto a las afectaciones al patrimonio por el crecimiento acelerado de la población.

Para realizar la intervención en el sitio arqueológico, se debe realizar un diagnóstico de daños y las causas que los generan, así como tomar en cuenta las opiniones de un equipo multidisciplinario para tomar las mejores decisiones para la intervención del sitio.

Posteriormente, se analiza el por qué la divulgación científica juega un papel importante en la conservación del patrimonio edificado y cómo pueden aprovecharse los avances tecnológicos como herramienta para conectar a la sociedad con los inmuebles patrimoniales.

\section{El sitio arqueológico de Los Padres y su arquitectura}

Los Padres, también llamado el Rehilete, es un sitio arqueológico representativo de las culturas del Valle de Atemajac, ubicado al poniente de la mancha urbana del AMG, a un costado del cerro del Colli dentro de la colonia 12 de Diciembre en el municipio de Zapopan.

Los Padres está ubicado sobre las calles prolongación Guadalupe y Volcán Quinceo. Según el plano del Registro Público de Monumentos y Zonas Arqueológicas del INAH (1985), se localizaron alrededor de veinticinco montículos en una superficie total de aproximadamente $100 \mathrm{Ha}$.

El polígono de protección perteneciente a Los Padres se vio afectado por el crecimiento de la población a las periferias de la ciudad, reduciéndolo a ocho hectáreas (de las $100 \mathrm{Ha}$ originalmente) y provocando la desaparición de más de la mitad de las estructuras, de las cuales se conservan alrededor de una decena de ellas (ver imagen 1).

El sitio pertenece a la fase Grillo (temporalidad propuesta por Javier Galván que corresponde al 450-90o d.C.) y está localizado al noroeste del sitio arqueológico El Iztépete, el más conocido en el Área Metropolitana de Guadalajara, y el único que actualmente se encuentra abierto al público. Debido a su cercanía, su temporalidad y a la relación entre los restos de zonas habitacionales, se cree que los sitios 


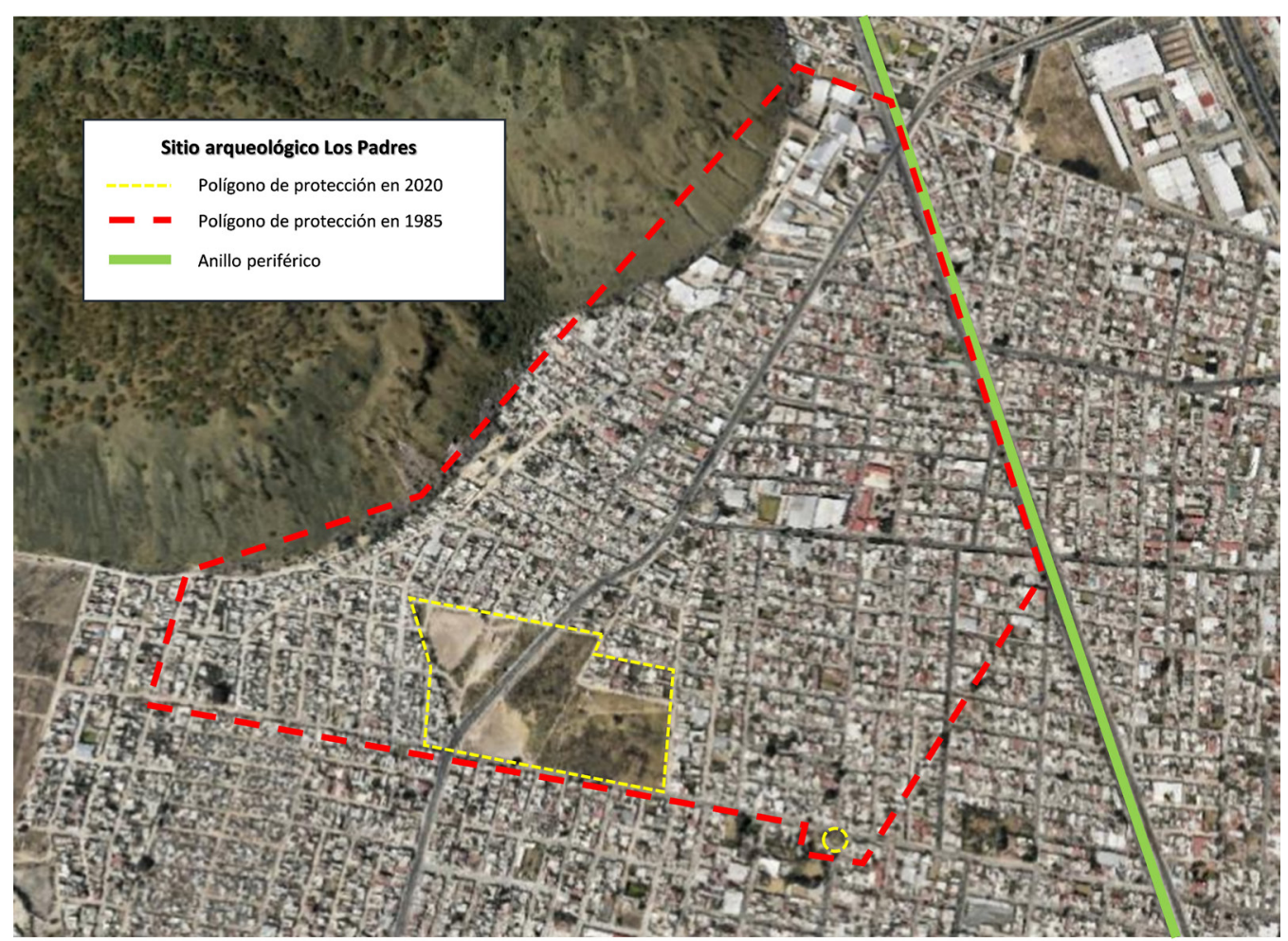

Imagen 1. Ubicación del polígono de protección de Los Padres. Gráfico del autor, fotografía satelital de Google Earth. de El Iztépete, Los Padres, Santa Ana Tepetitlán y El Tizate, todos sitios arqueológico ubicados en Zapopan, pertenecieron a un mismo conjunto durante la época prehispánica (Rubio, s.f.), convirtiendo a Los Padres en una extensión del centro ceremonial ubicado en el sitio arqueológico de El Iztépete o viceversa.

Se han encontrado dentro de tumbas de tiro pertenecientes a la fase Tabachines (700 a.C. al 450 d.C., más antiguas que los Padres), algunas maquetas de gran importancia para la arqueología del Occidente debido a que son muestras de la arquitectura que hoy se aprecia en ruinas y bajo una capa vegetal. Estas maquetas recrean las actividades humanas que se realizaban durante la época prehispánica y en ella se observa también el tipo de arquitectura, generalmente con cubiertas a cuatro aguas y decoraciones geométricas, que yacen sobre plataformas también decoradas con pigmentos (Fernández y Deraga, 2014). Esta característica se encuentra presente también en el Valle de Atemajac, la cual denota que las estructuras que vemos hoy, son solamente las bases de las edificaciones y no todo el conjunto prehispánico.

Durante la fase Grillo (450-90o d.C.), se desarrollaron estructuras de planta rectangular con ciertas características en sus elementos arquitectónicos muy similares entre otros sitios de la región Occidente mesoamericana, como el Bajío y los Altos de Jalisco (Beekman, 1996).

Esta característica de la región Occidente también se observa en el sitio arqueológico de El Iztépete. La amplia superficie de las estructuras ha hecho pensar a los investigadores que estas funcionaban solamente como bases y que existieron edificaciones sobre las mismas. Un ejemplo es el de la Estructura I, en el que Javier Galván realiza un análisis de este basamento, añadiendo una edificación en la superficie de la estructura para su recreación (Galván, 1975). 


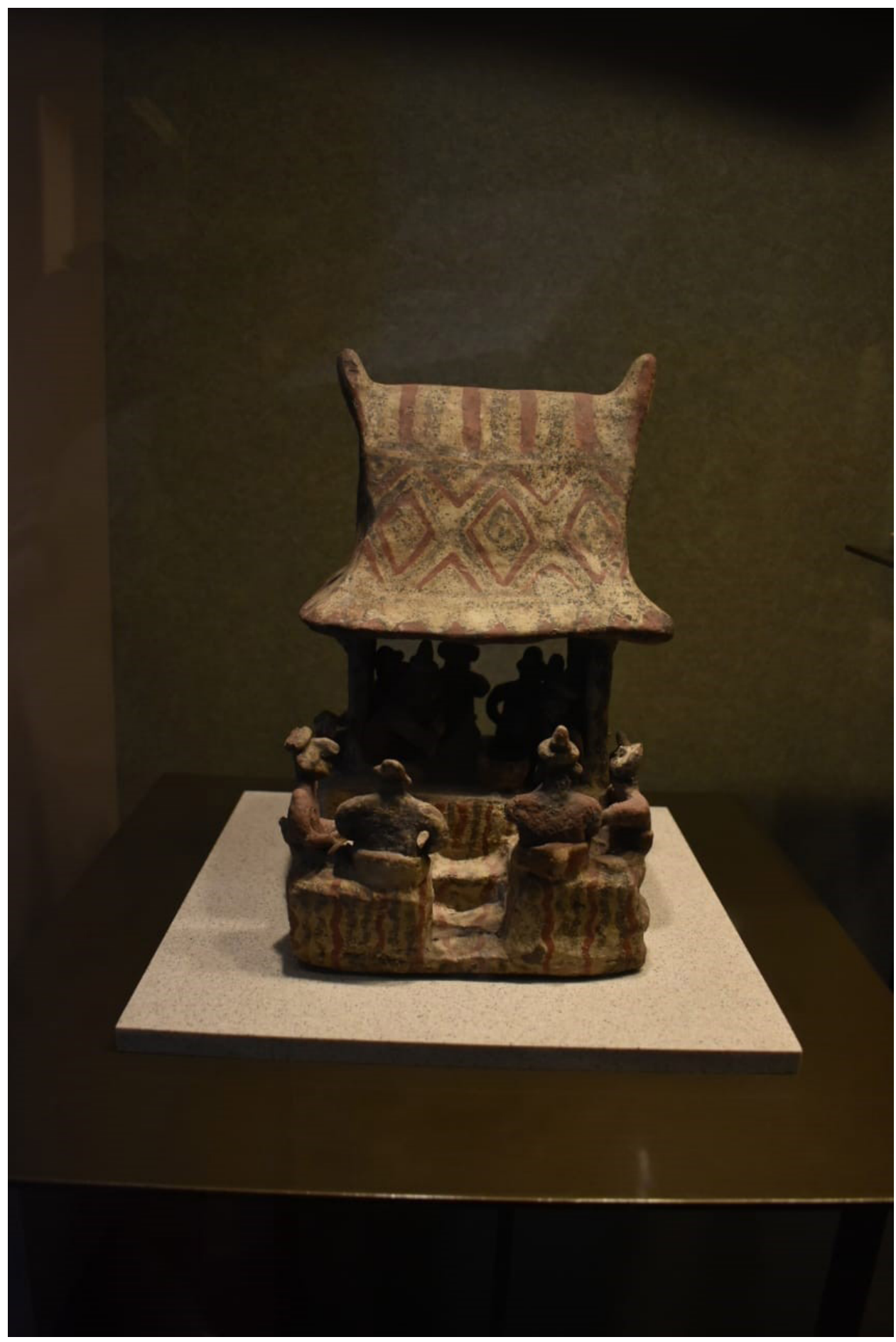

Imagen 2. Maqueta prehispánica de tumba de tiro.

Fotografía del autor, tomada en el Museo Nacional de Antropología. 


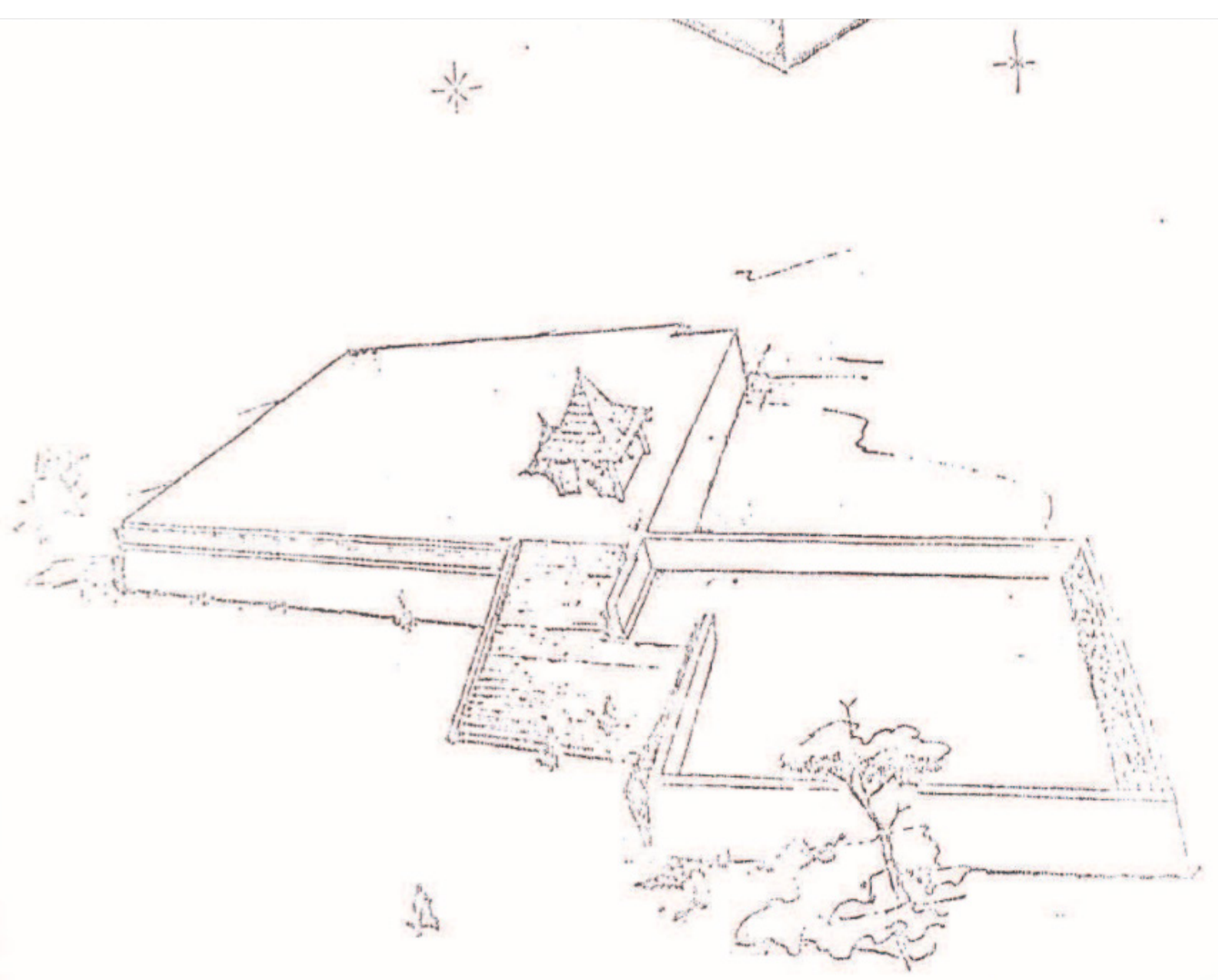

Imagen 3. Recreación hipotética de la Estructura I del sitio arqueológico El Ixtépete. Ejemplo de base y templo prehispánico. Fuente: Informe preliminar de las exploraciones efectuadas en la zona arqueológica de El Ixtépete, Javier Galván, 1973. 


\section{Diagnóstico del sitio}

El actual polígono del sitio arqueológico de Los Padres está delimitado por las calles Volcán Quinceo, San Juan, Nogal, Guayabo, Álamo y Laurel; así como la presencia de un montículo aislado en la glorieta ubicada entre las calles Volcán Quinceo y Puerto Mazatlán (Plan Parcial de Desarrollo Urbano ZPN-7 "El Colli”, s.f.), sin embargo, el crecimiento de la población dejó al sitio confinado dentro de la mancha urbana del AMG, provocando daños que lo han llevado a estar en riesgo de desaparecer.

Como se aprecia en la imagen cuatro, el polígono del sitio fue invadido por casi tres manzanas, y por la Prol. Av. Guadalupe, la cual divide el área de protección en dos. Actualmente, ambas partes se encuentran circundadas por una malla metálica y no es posible acceder al mismo sin permiso.

Lamentablemente, Los Padres presenta una problemática en cuanto a patologías urbanas, basta con visitar el sitio para identificar su estado de conservación. Una de estas, es el deterioro causado por el nulo mantenimiento del sitio arqueológico que afecta directamente a la imagen urbana de los alrededores. Este agente tiene características en el ámbito social debido a que los límites del sitio han sido utilizados como basurero por largo tiempo y su abandono ha generado

Imagen 4. Poligonal de protección del sitio arqueológico de Los Padres confinada dentro de la mancha urbana. Fuente: Fotografía satelital de Google Earth. inseguridad en la zona, incluyendo algunos pozos de saqueo.

Es notable la pérdida de carácter de zona arqueológica ya que el inmueble no es considerado por los habitantes de la zona como tal,

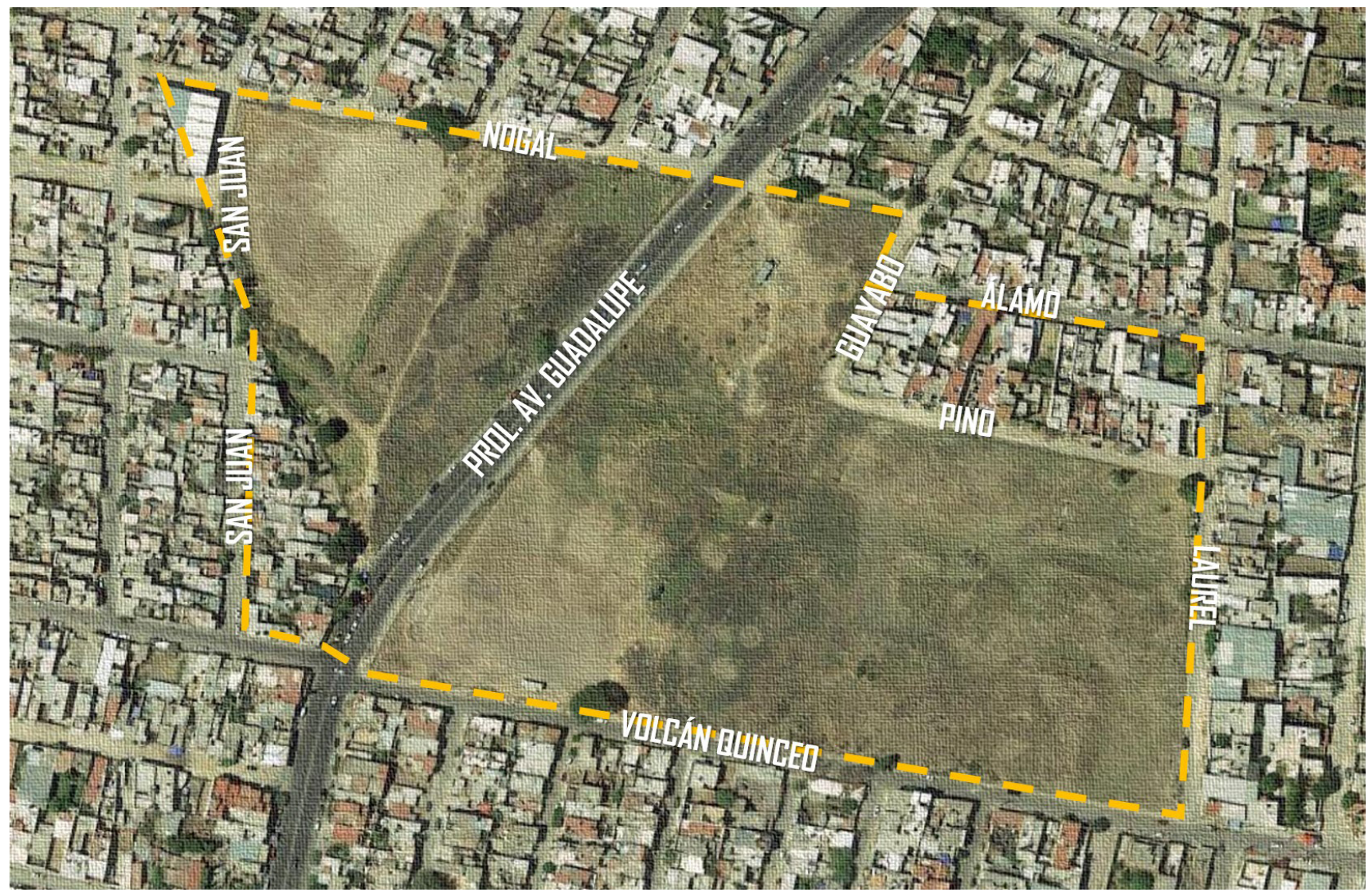




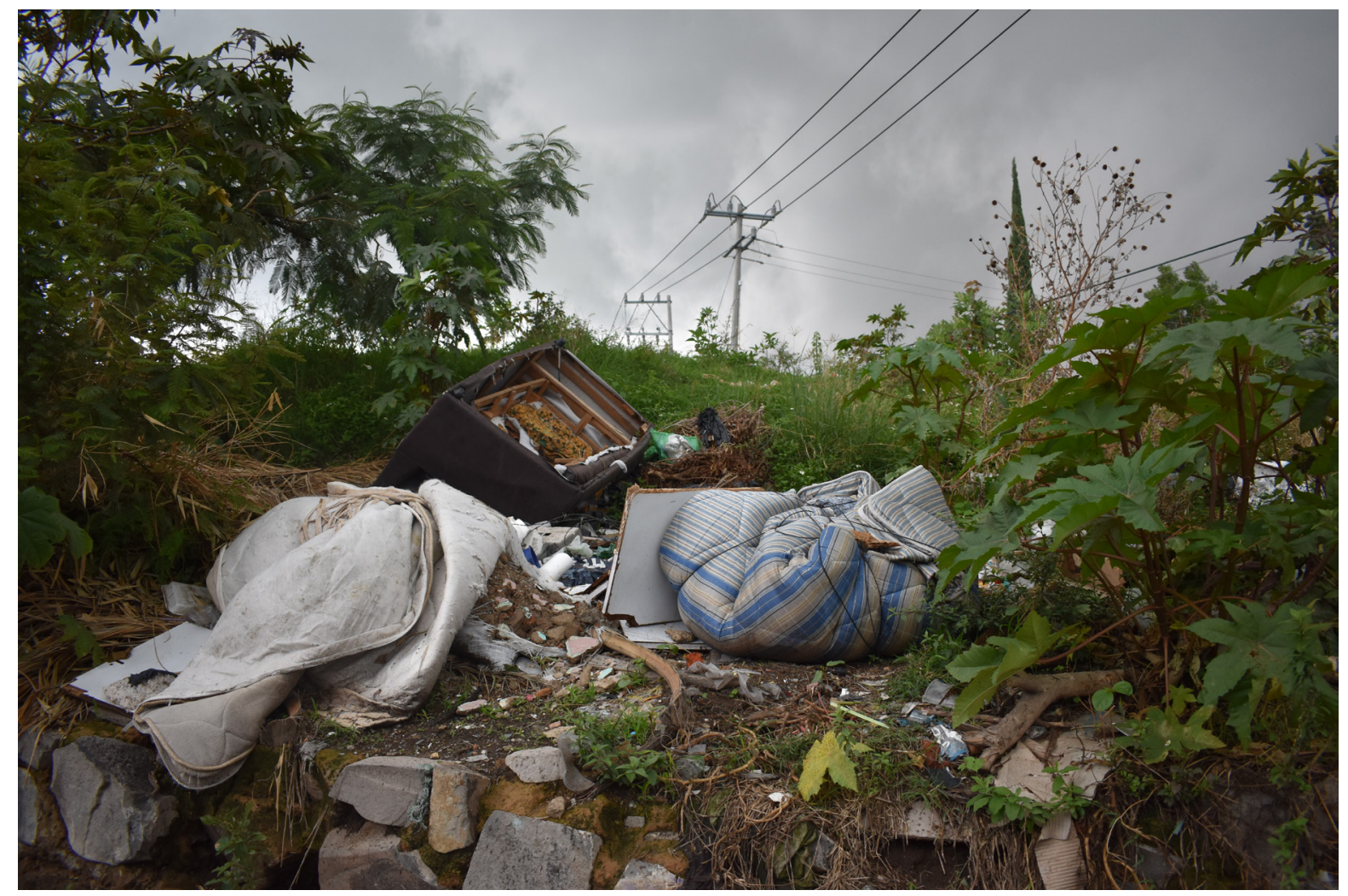

Imagen 5. Montículo de Los Padres. Se observa la presencia de basura. Fuente: del autor. Capturada el 8 de julio del 2020.

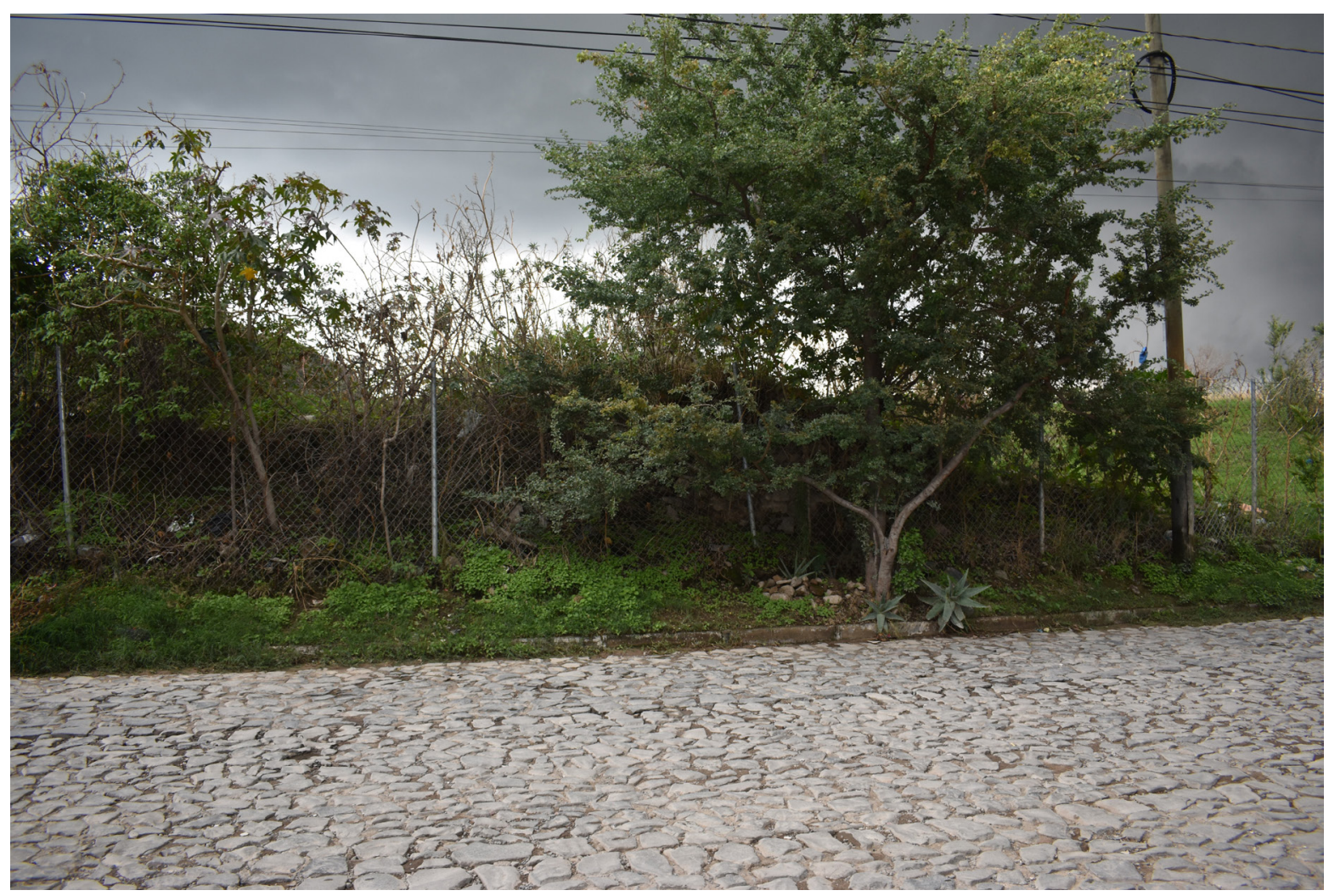

Imagen 6. Montículo rebanado de Volcán Quinceo. Fuente: del autor. Capturada el 8 de julio del 2020. 


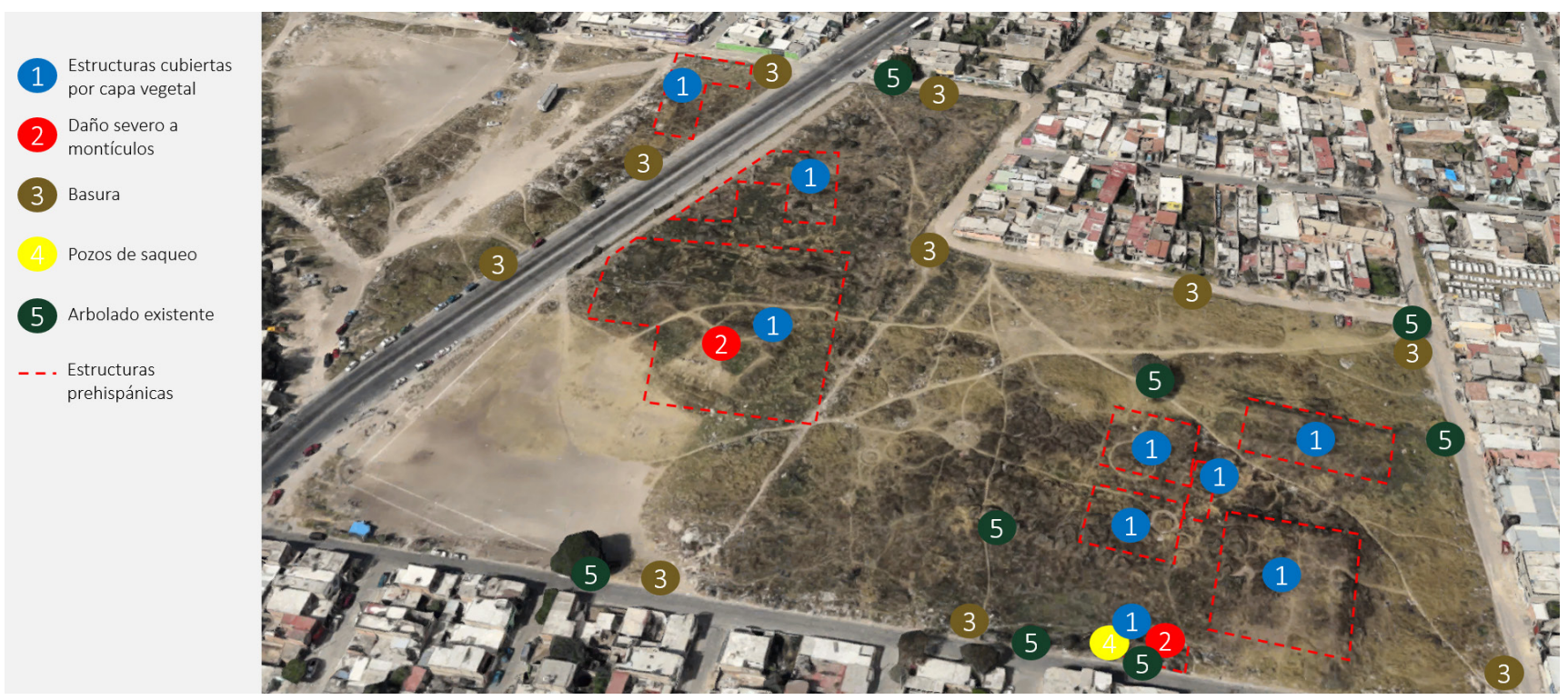

Imagen 7. Esquema del estado de conservación del sitio arqueológico de Los Padres. Fuente: propia. Basado en el plano de ubicación de montículos proporcionado por el arqueólogo Igor Quintana del centro INAH Jalisco). sino más bien es un baldío generador de inseguridad y de una gran cantidad de desechos.

La problemática acerca de la falta de divulgación a la sociedad es preocupante denotándose al realizar entrevistas a los vecinos. Muchos de ellos consideran un mito la presencia de estructuras prehispánicas en el predio, otros reconocen la posibilidad, pero desconocen completamente qué cultura habitó el sitio (algunos creen que fueron mayas) o qué es lo que se encuentra actualmente.

Es notable el abandono del inmueble, el tiempo ha generado deterioro debido a que la misma patología social causada por el incremento de la mancha urbana, poco a poco ha destruido lo que queda del sitio. Por la calle Volcán Quinceo, por ejemplo, se aprecia una estructura prehispánica que fue segmentada durante el trazo de la calle.

La prolongación avenida Guadalupe también ha invadido el área de protección del sitio arqueológico, dividiéndolo en dos y destruyendo lo que, según el plano del INAH, fueron tres montículos prehispánicos.

A continuación, se muestra un esquema con las afectaciones al sitio arqueológico de Los Padres mencionadas anteriormente:

Como se aprecia en el esquema y en las imágenes, la problemática es seria, sin embargo, afecta no sólo a este sitio arqueológico, sino a todos los que aún quedan dentro del AMG, incluyendo al único que se encuentra abierto al público: El Iztépete. Debido a ello, se pretende realizar una propuesta de intervención en el sitio que ayude a rescatar lo que aún queda del mismo, así como la propuesta de divulgación de la información a la sociedad, para que se dé a conocer el pasado prehispánico del Valle de Atemajac. 


\section{Caso Iztépete: sitio arqueológico en abandono}

El Iztépete es un sitio arqueológico ubicado en el municipio de Zapopan, Jalisco, a unos dos kilómetros al sureste de Los Padres, casi en el cruce de la Avenida Mariano Otero y el Anillo Periférico poniente Manuel Gómez Morín. Pertenece al conjunto urbano antes mencionado con Los Padres, Santa Ana Tepetitlán y El Tizate.

El sitio arqueológico cuenta con un núcleo principal conformado por tres estructuras principales, las cuales fueron liberadas de la capa vegetal que las cubría (aunque no completamente) y se realizaron los primeros trabajos de restauración en 1955 (Red Nacional de Información Cultural, 2020). Las estructuras fueron denominadas Estructura I, II y III dentro de una superficie de 6 ha, y zonas habitacionales de 36 ha en los alrededores (Gómez, 2001).

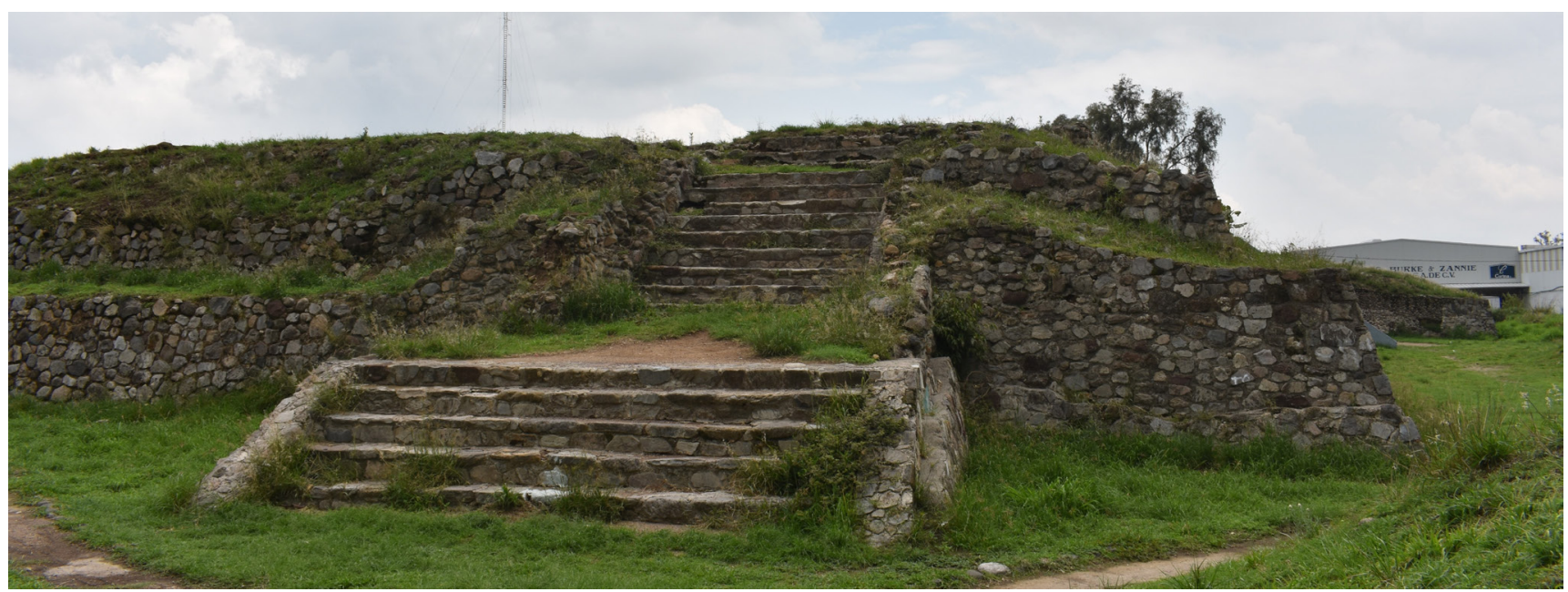

Imagen 8. Estructura I del sitio arqueológico El Ixtépete. Fuente: fotografía del autor. Capturada en julio del 2020.
La Estructura I es considerada la principal del sitio arqueológico, la cual consta de cinco etapas constructivas con elementos arquitectónicos no simétricos y una amplia superficie en la cima como puede observarse en la imagen 3. Lo anterior ha hecho pensar a los investigadores que pudo haber tenido varias edificaciones en la cima de la estructura construidos con materiales perecederos (Gil, 2007).

En el ingreso al sitio, se encuentra una caseta de vigilancia con un guardia de seguridad. El acceso es libre y no es necesario el registro, por lo que no hay una cifra exacta de la cantidad de visitantes que acuden al sitio ni de las personas que usan el sitio únicamente para cortar camino a sus destinos.

Es notable el abandono, la falta de mantenimiento y seguridad en el sitio, que se puede observar en el graffiti de los muros de las estructuras prehispánicas e incluso en la caseta de vigilancia.

A pesar de que está abierto al público y el acceso es gratuito, El Iztépete tiene bajo flujo de visitantes y su abandono lo ha dejado ex- 
puesto a vandalismo, saqueos y destrucción del patrimonio arqueológico del Valle de Atemajac.

El sitio no genera ingresos, por lo que las autoridades lo mantienen como actualmente se puede apreciar. Lo anterior se debe a la falta de divulgación en cuanto a patrimonio arqueológico, basta con preguntar a los habitantes del Área Metropolitana de Guadalajara para darse cuenta que la gran mayoría desconocen la existencia de este sitio arqueológico o de cualquier otro del área.

\section{Propuesta conceptual de intervención}

La propuesta consiste en la revitalización del área de protección delimitada en el Plan Parcial de Desarrollo Urbano ZPN-7 como PP-PH (Área de Protección del Patrimonio Histórico como lo marca el plan parcial de desarrollo urbano) del sitio arqueológico de Los Padres, en el que la población vecina al predio resulte beneficiada, tomando en cuenta las necesidades de la zona y los usos que actualmente se le dan al sitio dentro del perímetro de protección.

La revitalización consiste en la reutilización del predio otorgándole un uso como área verde, abierta y recreativa central (EV-C, como lo marca el Plan Parcial de Desarrollo Urbano), de una forma similar a la que se realizó en el estado de Yucatán con los restos de la antigua ciudad maya de T’Ho (una ciudad tan importante como Chichén Itzá o Uxmal) que también han quedado confinados dentro de la mancha urbana de la ciudad de Mérida (Ligorred, 2009).

En la intervención de T’Ho, se buscó la revitalización de los sitios por medio de la valoración y conservación del patrimonio arqueológico de Yucatán, tomando en cuenta la rentabilidad de la inversión pública y privada. Los proyectos yucatecos buscan el beneficio social mediante la creación de espacios verdes, públicos, didácticos, recreativos y deportivos, convirtiéndolos en un instrumento para la educación y la valoración social del patrimonio arqueológico de Mérida (Ligorred, 2005).

Se impulsó una red de parques arqueológicos en los que se realizaron proyectos de integración urbana en las áreas de protección arqueológicas, convirtiéndolas en espacios públicos y/o parques temáticos, buscando la participación ciudadana a través de los consejos vecinales, los cuales aseguraron la protección y vigilancia de los espacios. Además, se propuso la elaboración de planes de gestión que aseguren el mantenimiento y la difusión de los vestigios (Ligorred, 2009).

El proyecto de intervención de Los Padres pretende aplicar esta metodología tomando en cuenta el beneficio de la población y la valoración del patrimonio, es decir, que la sociedad reconozca el valor útil, lógico, estético y social de la obra arquitectónica (Villagrán, 1964) utilizando la divulgación como herramienta, por medio de actividades educativas dentro del predio con el fin de que la población 
en general conozca la importancia de la conservación del patrimonio arqueológico.

Para el proyecto, se pretende reanimar la zona que actualmente se encuentra deteriorada, evitando caer en un proyecto de intervención fallido como en caso del sitio arqueológico de El Iztépete. Es importante resaltar que, para la realización de la intervención, se deberá trabajar con un equipo interdisciplinario de profesionistas expertos en el tema.

Se propone realizar obras como la reutilización, conservación y valoración del patrimonio arqueológico, así como la divulgación de los hallazgos científicos para su preservación, por lo que el proyecto de intervención se dividirá en tres fases.

\section{Fase uno}

La primera fase consiste en el rescate del área de protección y la recuperación del predio desde el marco legal (debido a que tiene varios propietarios, aunque la mayor parte ya es propiedad de las autoridades). Esta fase incluye la recuperación de la totalidad del polígono de protección, identificando los asentamientos irregulares y todo lo que invada el área de protección.

$\mathrm{Su}$ recuperación debe realizarse por medio de una declaratoria que deberá ser expedida por el Presidente de la República (Reglamento de la Ley Federal sobre Monumentos y Zonas Arqueológicas, Artísticos e Históricos, 1975). Con ella, se debe lograr que los propietarios del área de protección lo den en concesión al Gobierno del Estado a cambio de una indemnización, ya que por ser propiedad federal el polígono debe ser expropiado (Ley General de Bienes Nacionales, 2004).

Una vez finalizada la recuperación total del polígono de protección, el sitio podrá ser vigilado, administrado y conservado por la Secretaría de Educación Pública, la cual, en conjunto con el Instituto Nacional de Antropología e Historia (INAH), podrán otorgar permisos o autorizaciones para la realización de actividades cívicas o culturales (Ley General de Bienes Nacionales, 2004).

Posteriormente, deberá gestionarse la inversión pública y privada y realizar estudios previos a la intervención directa, realizado por un grupo interdisciplinario de profesionistas, en el que se identificará la totalidad de las estructuras prehispánicas tanto las visibles en la prospección de superficie como las que no, con previa autorización del Instituto Nacional de Antropología e Historia. En esta fase, se propone el uso de herramientas tecnológicas como la prospección magnética o la utilización del Radar del subsuelo $3 \mathrm{D}$.

Finalmente, se realizará la interpretación conjunta de todos los estudios arqueológicos, con la presencia del equipo interdisciplinario. Lo anterior para identificar estructuras no visibles en el predio, por lo que la propuesta de intervención presentada podrá ser modificada después de la realización de este estudio. 
Imagen 9. Delimitación del polígono de protección de Los Padres (rojo) y las áreas recreativas de la zona (azul). No hay presencia de parques. Fuente: del autor, imagen satelital de Google Earth, 2020.

\section{Fase dos}

La fase dos consiste en la reutilización del polígono de protección al otorgarle un uso como área verde, abierta y recreativa con la posibilidad de adquirir usos culturales y educativos, como lo permite el Plan Parcial de Desarrollo Urbano ZPN-7, y usando como analogía las intervenciones en los sitios arqueológicos de Yucatán, sin embargo, el contexto urbano inmediato se encuentra en malas condiciones, por lo que se deberá intervenir de forma obligada.

Es importante mencionar que la zona, de uso habitacional predominante, carece de parques y/o áreas recreativas. Cuenta únicamente con una plaza cívica (1), una unidad deportiva (2) y un centro comunitario (3), que pueden apreciarse en la imagen 9, por lo que se añade un área verde a las necesidades de la población.

Otro punto importante es que actualmente el área es utilizada por los habitantes de la zona como canchas de baseball. Únicamente los domingos, el predio se abre a los habitantes de la zona y a un equipo visitante para jugar algunos partidos. Las familias de los jugadores los acompañan colocando sillas que llevan ellos mismos debajo del arbolado existente en cada cancha.

Es importante resaltar que esta actividad deportiva ha creado un vínculo emocional de los habitantes de la zona con el sitio, volviéndose una actividad que ha logrado la apropiación del mismo y que será una oportunidad para el proyecto de reutilización del sitio de unifi-

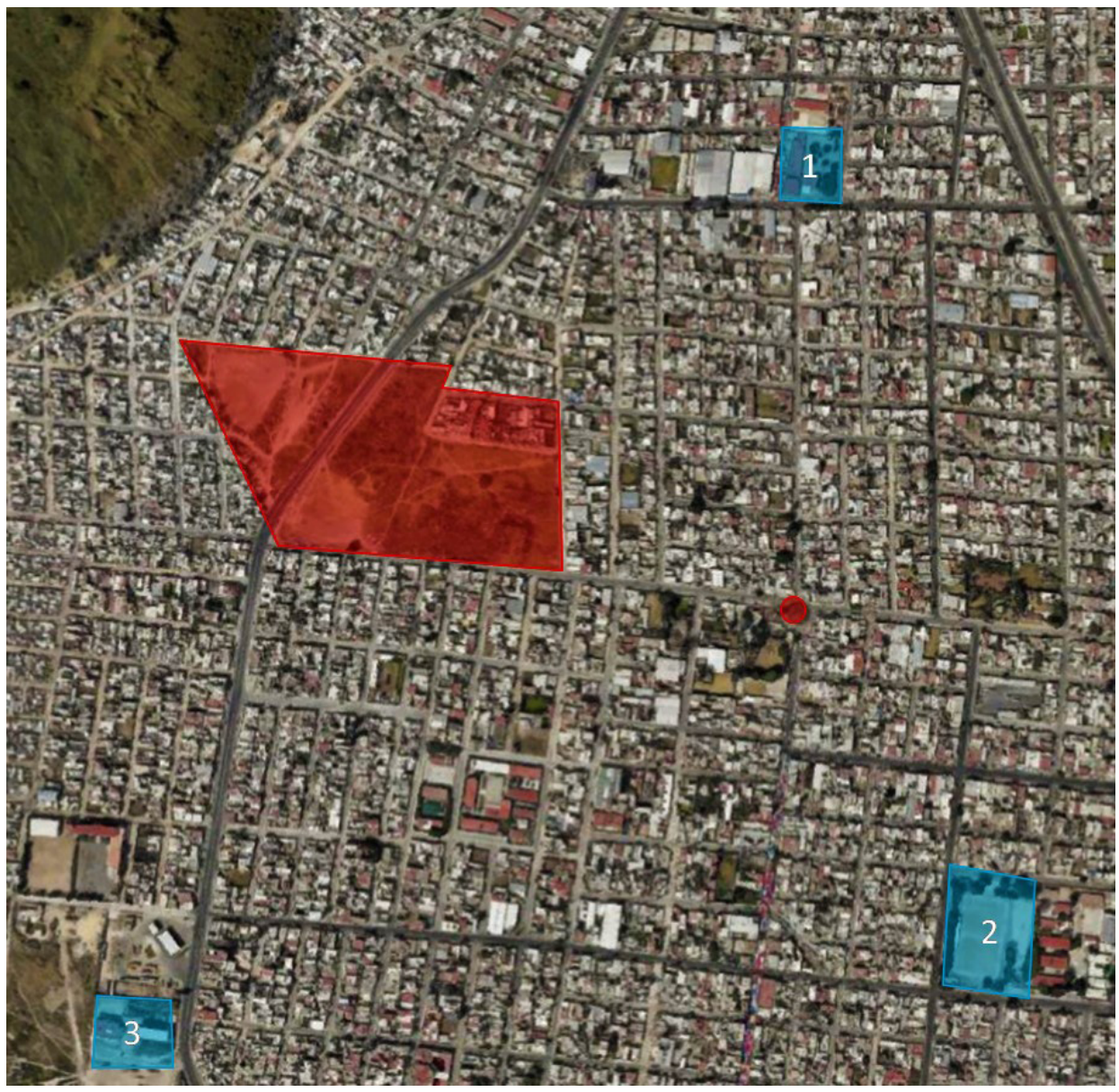


car un área cultural, un área deportiva y un área verde, siempre tomando en cuenta los estudios previos de la fase uno.

La valoración es uno de los puntos a considerarse como más importantes en esta propuesta, para no caer en un proyecto fallido como el caso de El Iztépete. Consiste en devolver el carácter de centro ceremonial prehispánico a través de la divulgación y prevenir futuros daños a las estructuras.

Es necesaria la participación de la sociedad en la conservación del patrimonio arqueológico (como se mostró en la analogía yucateca), por lo que la divulgación de la importancia de su conservación es un punto importante. Para ello, se proponen cédulas informativas con datos generales y un código QR en cada una de las estructuras, que lleven al usuario a una aplicación del sitio arqueológico, que contenga información detallada de las estructuras, la cual pueda ser modificada al mismo tiempo que se generen nuevos hallazgos e investigaciones del sitio.

Se proponen talleres educativos al aire libre con actividades como la implementación de la ludificación para niños y adultos por medio de herramientas tecnológicas como la realidad aumentada.

\section{Fase tres}

Imagen 10. Ubicación de canchas de baseball. Fuente: del autor, imagen satelital de Google Earth, 2020.

La tercera fase consiste en la restauración de las estructuras prehispánicas con el fin de hacer visibles los montículos al público. Es importante resaltar que la intervención en montículos arqueológicos, de-

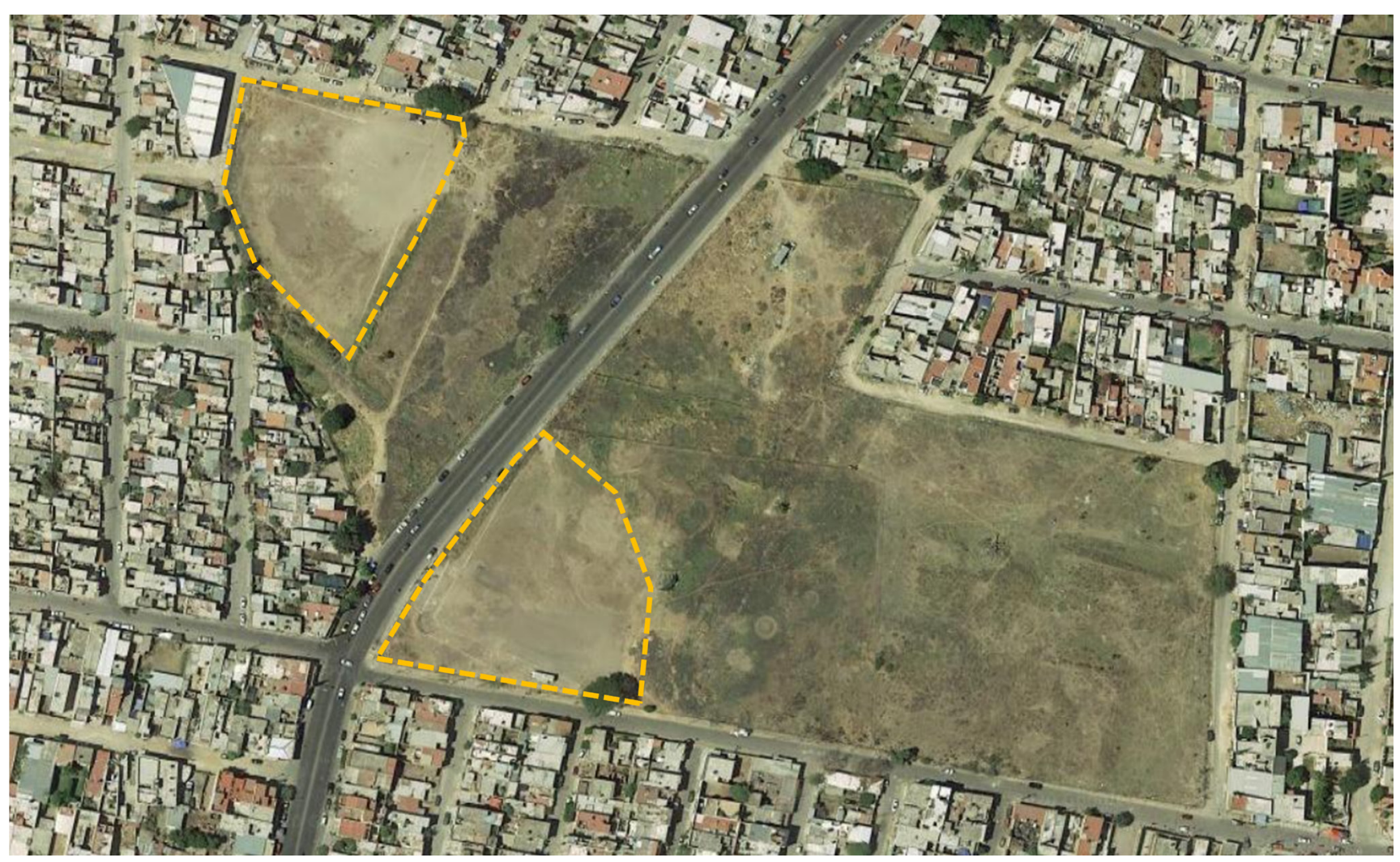


be ser realizada por la mano de miembros del Instituto Nacional de Antropología e Historia, especializados en arqueología del Valle de Atemajac, tal como lo especifica la Ley Federal sobre Monumentos y Zonas Arqueológicas, Artísticas e Históricas (1972).

Se deberá llevar a cabo la liberación de la capa vegetal que actualmente cubre los montículos prehispánicos, reintegrar los materiales que conforman la estructura y devolver a su estado original las estructuras, sin embargo, tomando en cuenta que la arquitectura de tierra requiere bastante mantenimiento y el abandono actual del patrimonio arqueológico del Valle de Atemajac, se puede proponer la consolidación de la estructura con una mezcla a base de cal para disminuir el mantenimiento, técnica que se ha utilizado en varios sitios arqueológicos como Teocaltitán en Jalostotitlán, Jalisco. Lo anterior sólo si lo decide un arqueólogo especializado y es aprobado por el INAH.

Lo anterior se resume en la siguiente imagen:

- Reutilización: en el que se le da un nuevo uso como espacio verde, abierto y recreativo. Actualmente el área se utiliza como cancha de baseball (sólo los domingos), por lo que se conservará el uso de la misma. Este uso puede ayudar a generar ingresos para el mantenimiento del sitio. Además, se agregan al proyecto áreas verdes.

- Conservación preventiva: medida contra vandalismo y saqueos al cerrar el sitio y otorgarle un único acceso. Se busca la participación ciudadana a través de los consejos vecinales, como en la analogía de T’Ho, los cuales aseguraren la protección y vigilancia del sitio arqueológico. Contratación de personal de limpieza, mantenimiento y prevención de deterioro de las estructuras.

- Valoración: un punto importante de la intervención en el que se devuelva el carácter de sitio arqueológico a través de la divulgación con herramientas tecnológicas.

- Restauración: Se propone la liberación de la capa vegetal que cubre los montículos, la reintegración de las piedras talladas que conforman las estructuras y la posible consolidación de la estructura. La restauración será realizada por el INAH y las decisiones finales serán tomadas por la institución.

Las fases uno y dos, puntos principales de esta propuesta, pueden llevarse a cabo sin la fase tres (la restauración de los montículos prehispánicos). Las estructuras pudieran permanecer cubiertas por la capa vegetal que las ha protegido hasta ahora y divulgar su existencia a través de herramientas como la realidad aumentada, sin embargo, es urgente la intervención del sitio. 


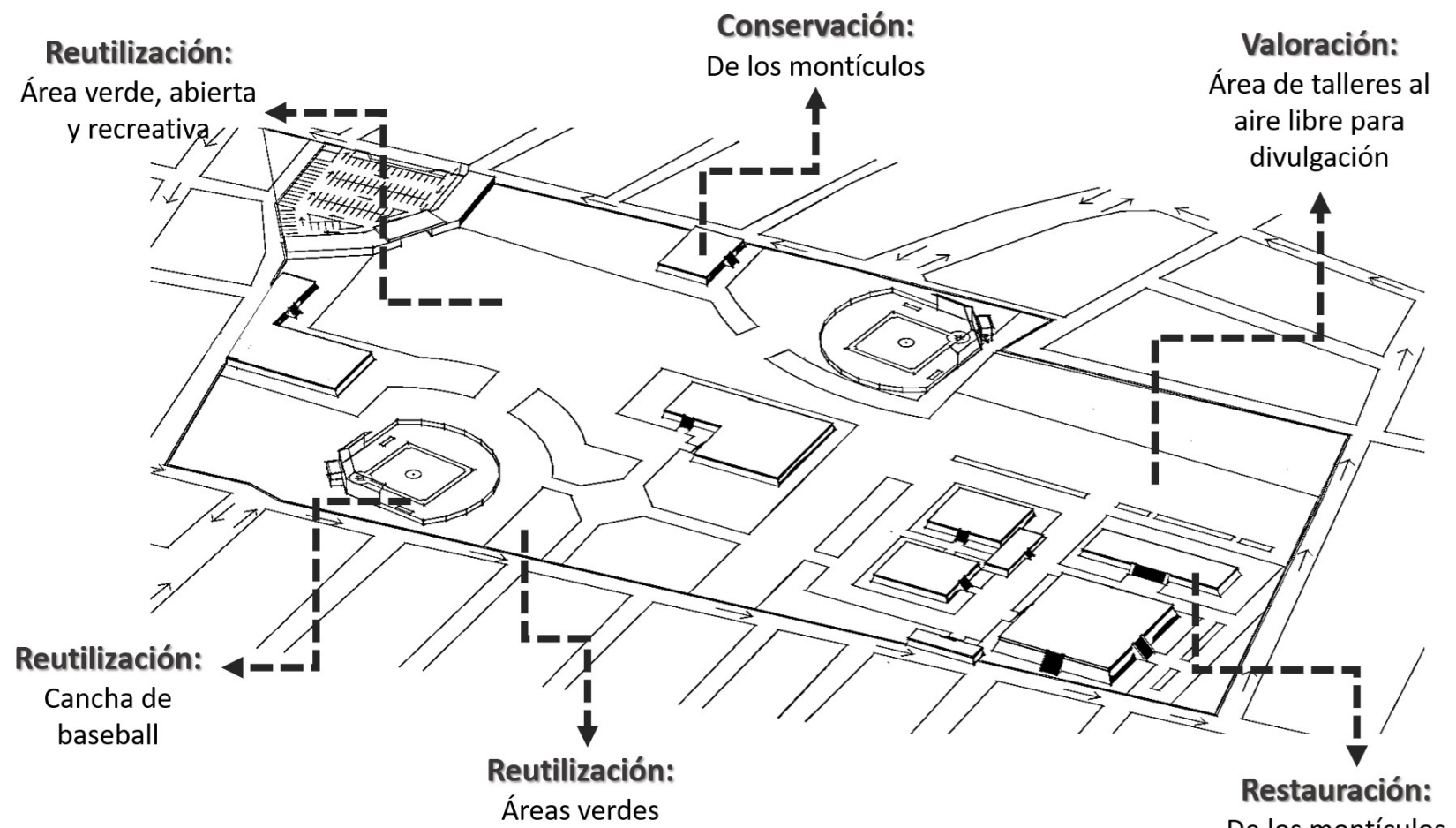

\section{La valoración a través de la divulgación}

Imagen 11. Propuesta de intervención en el sitio arqueológico de Los Padres. Fuente: del autor. Utilización del modelo de Luis Roberto González Velasco.
El caso de la falta de divulgación sobre la arqueología del Valle de Atemajac es un claro ejemplo de lo que Manuel Calvo Hernando menciona en su artículo: los avances científicos tienen un retraso respecto a la divulgación de la información, dando lugar a una gran diferencia entre el conocimiento de la comunidad científica y el público en general. Por ello, la divulgación es muy importante y debe verse como un elemento desarrollador del conocimiento de igualación cultural entre ambos (Calvo, 1997). Este retraso ha provocado el desinterés de la sociedad por la arqueología, dejando a los sitios en completo abandono. La divulgación científica de la arqueología del Valle de Atemajac pretende lograr en la sociedad un vínculo de apropiación con el espacio.

El comportamiento del ser humano hacia los espacios tiene que ver con la psicología de la apropiación, la cual consiste en que las personas, grupos y colectividades unan los procesos cognitivos, afectivos, de identidad y relacionales, es decir, los conocimientos, atracción por el lugar y su corresponsabilización dando lugar a un entorno apropiado para la sociedad (Vidal y Pol, 2005), procesos que llevan a las personas a hacer los sitios parte de su identidad:

A través de la acción sobre el entorno, las personas, los grupos y las colectividades transforman el espacio, dejando en él su "huella", es decir, señales y marcas cargadas simbólicamente. Mediante la acción, la persona incorpora el entorno en sus procesos cognitivos y afectivos de manera activa y actualizada. Las 
Imagen 12. Propuesta de intervención en el sitio arqueológico de Los Padres. Fuente: propuesta propia. Render realizado por Lilian Janette Rodríguez Cisneros y Angélica Muñoz Pérez de JM Arquitectos. acciones dotan al espacio de significado individual y social, a través de los procesos de interacción. (Vidal y Pol, 2005, p. 283).

Mediante los distintivos que deja la sociedad que conoce el lugar, esta reconoce el entorno y lo hace parte de su identidad, lo que genera acciones y define el comportamiento de las personas en el lugar, generando interés en la sociedad por el cuidado y mantenimiento del mismo. Debido a esto, en esta propuesta se considera de gran importancia la divulgación científica, con el fin de que la sociedad genere un vínculo con el sitio arqueológico y participe en su conservación.

Para llevar a cabo la divulgación, se propone el uso de herramientas como la realidad aumentada (RA), definida como "aquella que permite al usuario ver en todo momento el mundo real, al que se le superponen objetos virtuales coexistiendo ambos en el mismo espacio" (Ruiz, 2011, p. 215) y ha estado presente en la actualidad a través de aplicaciones que, como dice su definición, permiten superponer objetos en el mundo real y han ayudado a museos, escuelas, entre otros, a facilitar la comprensión de la información (Ruiz, 2011).

A diferencia de la realidad virtual (RV), en la cual el usuario queda inmerso en un entorno dominantemente virtual al que se le añaden objetos tanto reales como virtuales; en la realidad aumentada domina el entorno real al que se le agregan objetos virtuales (Heras, 2004).

Las estructuras prehispánicas del Valle de Atemajac que vemos hoy tanto en Los Padres como en El Iztépete, son solamente las bases de todo el conjunto, un dato que la comunidad científica conoce, pero el público en general no. Por ello, se propone la utilización de una aplicación de RA. Ésta permitirá sobreponer las edificacio-

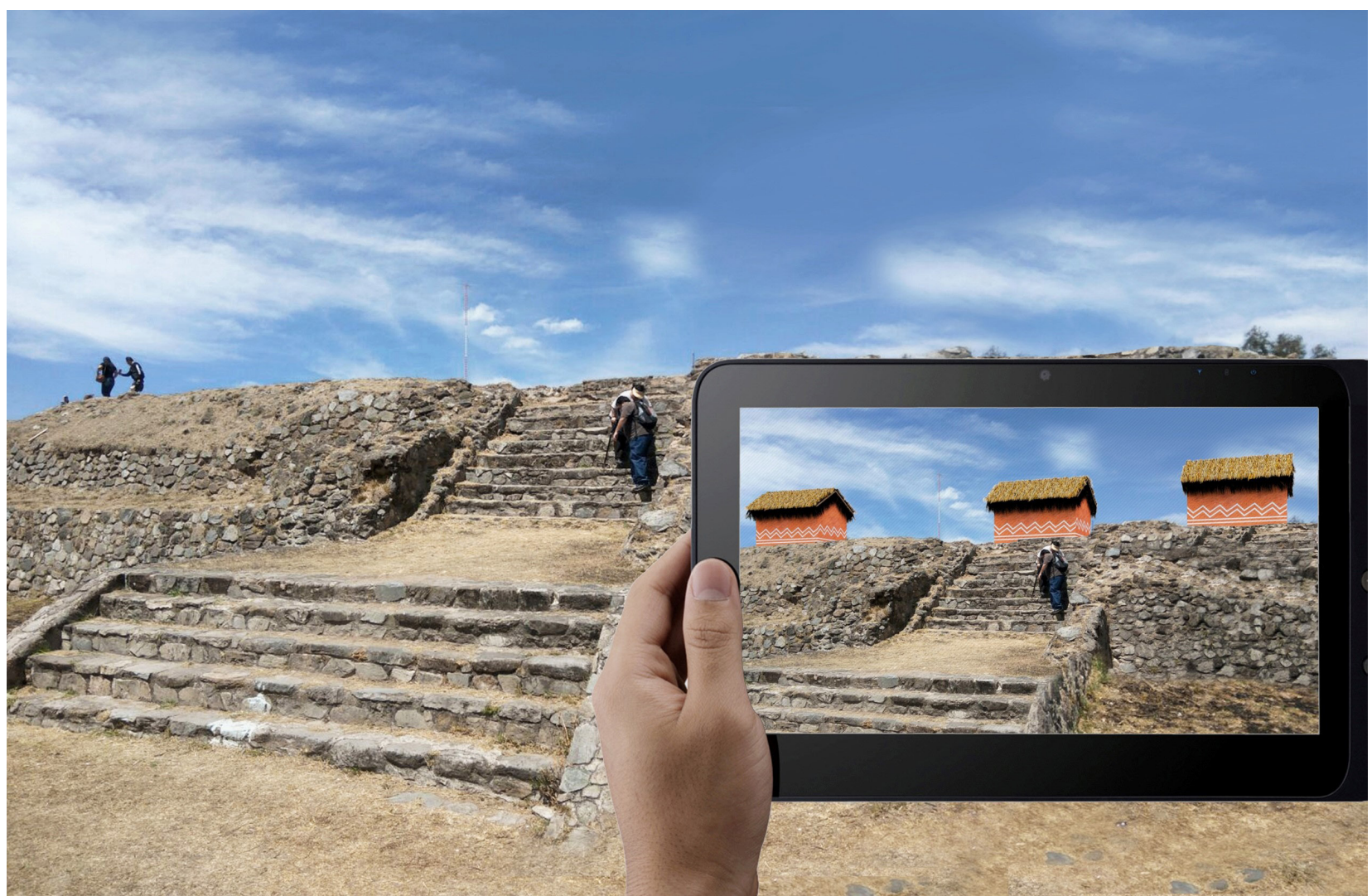


nes prehispánicas que ya no existen en un entorno real, tal como se muestra a continuación:

La utilización de esta aplicación, facilitará la comprensión de la arqueología del Valle de Atemajac para el público en general, la cual funciona con la combinación de una imagen de la estructura prehispánica real con una imagen virtual que recrea lo que ya no existe.

Podrá funcionar como un elemento de ludificación para involucrar al usuario de manera amena en el conocimiento sobre la arqueología del Valle de Atemajac y con ella crear una vinculación con él para transmitir información.

La ludificación (más conocida como gamificación), se define como la «utilización de algunos elementos y mecanismos propios de un juego, como la puntuación o el establecimiento de niveles que deben superarse, en un entorno no lúdico» (Oxford Languages, s.f.), que puede utilizarse como una herramienta de aprendizaje con el fin de motivar al usuario a acercarse al conocimiento científico por medio de dinámicas (narrativa), mecánicas (retos, competencias) y componentes (logros, puntos, niveles) (Ortiz, Jordán y Agredal, 2018).

Con ayuda de la ludificación, la realidad aumentada y el área de talleres al aire libre se pretende reforzar el aprendizaje del visitante. De esta manera, la divulgación dará lugar a un balance entre el investigador y el público en general.

\section{Conclusiones}

Son nueve los sitios arqueológicos registrados ante el Instituto Nacional de Antropología e Historia en el municipio de Zapopan que se encuentran en las mismas o más desfavorables condiciones que el sitio arqueológico de Los Padres. La mayoría de los sitios ya fueron alcanzados por la mancha urbana del Área Metropolitana de Guadalajara, producto del crecimiento acelerado de la población.

La propuesta presentada en este documento fue realizada con el fin de informar sobre el estado de conservación de los sitios y la urgencia de intervenirlos. Además, se pretende que el concepto de esta propuesta de intervención pueda ser aplicado de la misma manera a los nueve sitios arqueológicos de Zapopan, creando espacios verdes, abiertos y recreativos que beneficien a los vecinos de las zonas arqueológicas con áreas verdes de las que carecen, donde incluso puedan impartirse talleres sobre la importancia de la conservación del patrimonio arqueológico y utilizar los avances tecnológicos como herramienta de aprendizaje.

Aún falta mucho por descubrir sobre la arqueología del Valle de Atemajac, pero es importante que los avances científicos progresen al mismo tiempo que la información proporcionada a la sociedad, ya que son los vecinos de los sitios arqueológicos, los que cuidarán de los mismos una vez que se logre la apropiación del espacio. 


\section{Bibliografía}

Beekman, C. (1996). El Complejo el Grillo del centro de Jalisco: una revisión de su cronología y significado. En Williams, E. y Weigand, P. (eds.), Las Cuencas del Occidente de México. Época Prehispánica (pp. 247-291). Zamora: El Colegio de Michoacán.

Calvo Hernando, M. (1997). Objetivos de la divulgación de la ciencia. Chasqui. Revista Latinoamericana de Comunicación, (60), 38-42. Recuperado de <https://revistachasqui.org/index.php/chasqui/ article/view/1153/1182 el 28 de septiembre del 2020>.

De la Rosa Falcón, G. (1979). Restructuración del Templo Parroquial de Nuestra Señora de Guadalupe, Romita Guanajuato (Tesis de maestría). Escuela Nacional de Restauración, Conservación y Museografía, Ciudad de México, México.

Fernández, R. y Deraga, D. (2014). La zona occidental en el Clásico. En Manzanilla, L. y López Luján, L. (eds.), Historia antigua de México. El horizonte Clásico. Vol. II (pp. 161-201). Ciudad de México: Porrúa.

Galván, J. (1975). Informe preliminar de las exploraciones efectuadas en la zona arqueológica de 'El Ixtépete', Jal. Durante el mes de mayo de 1973. En Xiri Mesa de la Sociedad Mexicana de Antropología. Balance y perspectiva de la antropología de Mesoamérica y del Centro de México. Mesa redonda llevada a cabo en Xalapa.

Gil Flores, J. y Saenz, C. (2007). El Ixtépete: zona arqueológica, estudios y exploraciones. Guadalajara: Guías IJAH.

Gómez Gastelum, L. (2001). Cacicazgos prehispánicos en el Valle de Atemajac, Jalisco: Una sintesis de la arqueología de Tabachines, Bugambilias y El Iztépete. Guadalajara: Gobierno del Estado de Jalisco.

Heras Lara, L. (2004). La realidad aumentada: una tecnología en espera de usuarios. Revista Digital Universitaria, 5(7), 1-9.

Ley General de Bienes Nacionales. Cámara de Diputados del H. Congreso de la Unión, México, 2004.

Ligorred i Perramon, J. (2005). Arqueología, Desarrollo Urbano y Equipamiento Cultural en Mérida, Yucatán (México). En Paredes Guerrero, B. (ed.), Seminario Nacional de Conservación del Patrimonio Edificado: Experiencias de Adecuación para el Equipamiento Cultural (pp. 87-95). Mérida: Universidad Autónoma de Yucatán.

Ligorred i Perramon, J. (2009). Valoración y uso social del patrimonio arqueológico de Mérida, Yucatán. En Salazar González, G. 
(coord.), Modernidad, patrimonio, tecnología y diseño (pp. 406420). San Luis Potosí: Universidad Autónoma de San Luis Potosí.

Ortiz-Colón, A., Jordán, J. y Agredal, M. (2018). Gamificación en educación: una panorámica sobre el estado de la cuestión. Pesqui, (44) 1-17.

Oxford Languages (s.f.). Ludificación. En Diccionario Oxford Languages. Recuperado el 9 de noviembre del 2020.

Plan Parcial de Desarrollo Urbano Distrito Urbano ZPN-7 «El Colli». Gobierno de Zapopan (s.f.). Recuperado de <http://www.zapopan.gob.mx/transparencia/obras-publicas/distrito-zpnoz/>.

Red Nacional de Información Cultural (2020). Ixtépete. Zapopan: Sistema de Información Cultural. Recuperado de $<$ https://sic.cultura.gob.mx/ficha.php?table=zona_arqueologica\&table_id=55>.

Reglamento de la Ley Federal sobre Monumentos y Zonas Arqueológicas, Artísticos e Históricos. Cámara de Diputados del H. Congreso de la Unión, México, 1975.

Rubio Navarro, H. (s.f.). Pasado prehispánico de Guadalajara: La zona arqueológica El Iztépete (Tesis de licenciatura). Universidad de Guadalajara, Guadalajara, México.

Ruiz Torres, D. (2011). Realidad Aumentada, educación y museos. Revista ICONO14. Revista científica de Comunicación y Tecnologías emergentes, 9(2) 212-26. Recuperado de $<$ https://www.researchgate.net/publication/277216861_Realidad_Aumentada_educacion_y_museos>.

Vidal Moranta, T. y Pol Urrútia, E. (2005). La apropiación del espacio: una propuesta teórica para comprender la vinculación entre las personas y los lugares. Anuario de Psicología, 36(3), 281-297. 